\title{
Documentation of flora of Rara lake and adjoining areas in mid-western region of Nepal
}

\begin{abstract}
B. K. Basnet ${ }^{1}$
Rara National Park is the smallest national park of the country. It is rich in floral and faunal diversity. Rara is one of the sacred lakes and is listed as a Ramsar site. The aim of the study was to compile the representative flora of Rara lake and to present status of available vegetation. The research used both primary and secondary sources of data. Field visit was conducted in June, 2010 during which more than 300 plant specimens were collected. The secondary data were collected from Rara and adjoining area like Gamgadi. These data were thoroughly analyzed to understand the composition of vegetation. The study revealed the existence of about 224 flowering plant species in the area, under 173 genera and 67 families. Compositae was found to be the largest family ( 21 species and 17 genera) followed by Rosaceae (19 species and 10 genera).
\end{abstract}

\section{Key words: Rara lake, Ramsar site, floral composition, alpine flora, flowering plants}

$\mathbf{M}$ ugu district lies in Karnali zone in the midwestern development region of Nepal. It is inaccessible due the lack of motorable road. There is a newly established airport at Talcha which is about two hours journey from Rara lake and Gamgadi, the district headquarters of Mugu. There is regular flight from Nepalgunj and Surkhet, the regional headquarters of the mid-western region of Nepal. The main attraction of Mugu district is the Rara National Park (RNP). The park was established in 1976 with an objective of protecting the unique flora and fauna of the Humla-Jumla Region of Nepal. It was also declared as Ramsar site (number 1695) in 2007. The park covers an area of $106 \mathrm{~km}^{2}$ as core area and $197.76 \mathrm{~km}^{2}$ as buffer zone. The park ranges in elevation from $1,800 \mathrm{~m}$ to $4,039 \mathrm{~m}$ at Chuchemara Peak on the southern side of Rara Lake. On the northern side, the peaks of Ruma Kand and Malika Kand exist (Bhuju et al., 2007).

Despite being the country's smallest national park, RNP encloses the largest lake of Nepal (area: 10.8 $\mathrm{km}^{2}$, depth: $167 \mathrm{~m}$ ). The lake belongs to alpine freshwater category and lies at an altitude of 2,990 m. It is oval-shaped with an east-west axis. It has a length of $5 \mathrm{~km}$ and a width of $3 \mathrm{~km}$, and drains into MuguKarnali River via Nijar Khola.

The physiography of the study area comprises transHimalayan range covering entirely the temperate and sub-alpine flora. Mountains above 4,000 m runs along west to east with different patches of vegetation composition in different aspects around Rara lake.

The botanical exploration of Karnali zone started with the visit of Col. Bailey in 1956. He visited different parts of Karanali zone including Rara lake for botanical collection. Fifteen years later, British scientists gathered a large number of plants from this area. Stainton travelled the major parts of the zone in 1963 to explore its flora. Similarly, Itoh and Rajbhandary in 1965, and other Nepalese botanists also visited the zone on different occasions and assembled a large number of plants for herbarium, garden and other laboratory purposes (Manandhar, 1984). Ferro who visited it in 1979 has compiled limnological and biological data. Morphological, physical, chemical and biological information of Rara lake has already been published by Tokio and Yashiro in 1986.

\section{Materials and methods}

The research is based on both primary and secondary data. For the primary information, a field visit was conducted in June, 2010 during which, more than 300 plant specimens under different families were collected as herbarium specimens, with collection number, locality and plant habit. The secondary data was based on herbaria collected from Rara Lake and adjoining areas by different collectors at different times, and deposited by them at National Herbarium

\footnotetext{
${ }^{1}$ Department of Forest Research and Survey, Babarmahal, Kathmandu

Email: basnet_b@hotmail.com
} 
(KATH). Such data were further analyzed in accordance with family, generic and specific names.

The collected specimens were thoroughly examined and identified by using relevant references as Hooker (1883-1887), DPR (1994), Polunin and Stainton (1984). They were crosschecked with the herbarium specimens deposited at KATH. The nomenclature given in latest taxonomic literatures (Press et al., 2000; DPR, 2001) were adopted. The herbarium specimens are deposited at National Herbarium KATH, Godawari, Lalitpur, Nepal.

\section{Results and discussion}

The flora of the study area comprised a total of 224 species, 173 genera and 67 families of flowering plants (Annex 1). The research found Compositae to be the largest family (21 species under 17 genera) followed by Rosaceae (19 species under 10 genera), and Labiatae. They are followed by Graminae (14 species under 11 genera), Cyperaceae (13 species and 9 genera) and Umbelliferae ( 9 species under 7 genera). Twenty nine families were counted as least species number holding family. These included, Acanthaceae, Amaranthaceae, Asclepiadaceae, Balasminaceae, Buxaceae, Campanulaceae, Cannabinaceae, Coriariaceae, Dioscoreaceae, Dipsacaceae, Holoragaceae, Juglandaceae, Lentibulariaceae, Loranthaceae, Menyanthaceae, Onagraceae, Orchidaceae, Oxalidaceae, Phytolacaceae, Plantaginaceae, Primulaceae, Rubiaceae, Rutaceae, Tamaricaceae, Taxaceae, Thymelaceae, Urticaceae,Vitaceae and Zigiberaceae. Other 32 families had low number of species ranging from having two to eight species in each family. The family names followed by genus and species' names are arranged alphabetically (Annex 1).

Regarding the diversity of the species in the surrounding area, least number of species was observed under pine forests, especially in the planted area near Milli Chaur. High number of herbaceous plant was found in Lower Milli along the side of pasture land designated by the Park. Although collection of aquatic macrophyte remained the focus of interest during the field study, only a few numbers of such species could be collected because of difficult field situations. Myroiphylum spicatum under the family Holoragaceae, Urticularia australis under the family Lentibulariaceae and three species of Potamogeton were recorded during the field survey. In addition, species like Isolepis setacea under the family Cyperaceae and Rumex under the family Ploygonaceae were observed in semi-aquatic habitats.

More detailed survey of vegetation is required in order to deeply understand the availability of endemic and other endangered plant species. It is suggested that a comprehensive research be conducted in future in collaboration with academic universities within the budgetary frame of the Department of National Parks and Wildlife Conservation.

\section{Acknowledgements}

I express my gratitude to Mr. Sahas Man Shrestha, Director General, Department of Forest Research and Survey for his encouragement to conduct this study. I am grateful to Mr. Ramesh Shakya, Mr Dipendra Pokharel and Mr. Atul Man Joshi for their initiative to provide me the grant and necessary arrangement during the field visit. I am also thankful to the Nepal Army personnel and park staff for their support in providing medical services during the period of my illness. I express my sincere thanks to senior taxonomist Dr. Keshab Raj Rajbhandari for his valuable instruction during the identification of the collected specimen.

\section{References}

Bhuju, U. R., Shakya, P. R., Basnet, T. B. and Shrestha, S. 2007. Nepal Biodiversity Resource Book (Protected area, Ramsar site and World heritage Sites). ICIMOD and MOEST, Kathmandu, Nepal.

DPR. 2001. Flowering Plants of Nepal (Phanerogams). Bulletin of Department of Plant Resources No 18, Kathmandu, Nepal.

DPR. 1994. Enumeratin of Vascular plants of West Nepal. Bulletin of Department of Plant Resources No. 12, Kathmandu, Nepal.

Ferro, W. 1979. Some limnological and biological data from lake Rara. Journal of Nepal Research Centre $\mathbf{2}$ (3): 241-261.

Hooker, J.D. 1883-1887. Flora of British India. Vol. I - VII, Reev and Company, London, UK. 
Manandhar, N.P. 1984. A contribution to the flora of Jumla District and its environ, Nepal. J. Econ. Taxon. Bot. (India) 5 (3): 547-571.

Press, J. R., Shrestha, K. K. and Sutton, D. A. 2000. Annoted Checklist of the Flowering Plants of Nepal. Natural History Museum, London, U.K.

Polunin, O. and Stainton, A. 1984. Concise Flowers of the Himalaya. Oxford University Press. New Delhi, India.

Annex 1: List of the family wise species and genera

\begin{tabular}{|c|c|c|c|c|}
\hline S.No. & $\begin{array}{l}\text { Collection } \\
\text { number }\end{array}$ & $\begin{array}{l}\text { Name of } \\
\text { collector }\end{array}$ & Scientific Name & Family name \\
\hline 1. & 0104 & Basnet, B.K. & Aechmanthera gossypina (Wall.) Nees & Acanthaceae \\
\hline 2. & 0069 & Basnet, B.K. & Cyathula tomentosa (Roth) Moq. & Amaranthaceae \\
\hline 3. & 0113 & Basnet, B.K. & Arisaema flavum (Forsk.) Schott & Araceae \\
\hline 4. & 0114 & Basnet, B.K. & Acorus calamus L. & Araceae \\
\hline 5. & 0219 & Basnet, B.K. & Arisaema consanguineum Schott & Araceae \\
\hline 6. & 0002 & Basnet, B.K. & Vincetoxicum hirundinaria Medicus & Asclepiadaceae \\
\hline 7. & P14 & Basnet, B.K. & Impatiens laxiflora Edgew. & Balasminaceae \\
\hline 8. & 0003 & Basnet, B.K. & Berberis chitria Lindl. & Berberidaceae \\
\hline 9. & P005 & Basnet, B.K. & Berberis koehneana C.K. Schneid. & Berberidaceae \\
\hline 10. & 0112 & Basnet, B.K. & Betula alnoides Buch.-Ham. Ex D.Don & Betulaceae \\
\hline 11. & 0115 & Basnet, B.K. & Alnus nitida (Spach) Endl. & Betulaceae \\
\hline 12. & 0110 & Basnet, B.K. & Buxus polystachya & Buxaceae \\
\hline 13. & 0004 & Basnet, B.K. & Codonopsis & Campanulaceae \\
\hline 14. & 0118 & Basnet, B.K. & Cannabis sativa Linn & Cannabinaceae \\
\hline 15. & 0005 & Basnet, B.K. & Triosteum himalayanum Wall. & Caprifoliaceae \\
\hline 16. & 0047 & Basnet, B.K. & Lonicera angustifolia Wall & Caprifoliaceae \\
\hline 17. & 20674 & Basnet, B.K. & Lonicera quinquelocularis Hardw. & Caprifoliaceae \\
\hline 18. & 206741 & Basnet, B.K. & Lonicera obovata Royle & Caprifoliaceae \\
\hline 19. & P008 & Basnet, B.K. & Lonicera acuminata Wall & Caprifoliaceae \\
\hline 20. & P008 & Basnet, B.K. & Lonicera lancelata Wall & Caprifoliaceae \\
\hline \multirow[t]{2}{*}{21.} & P008a & Basnet, B.K. & Lonicera spinosa Jaquem. Ex Decne.) & \\
\hline & & & Walp. Wall & Caprifoliaceae \\
\hline 22. & 0006 & Basnet, B.K. & Arenaria sikiimensis Majumdar & Caryophylaceae \\
\hline 23. & 0168 & Basnet, B.K. & Gypsophylla cerastioides D.Don & Caryophylaceae \\
\hline \multirow[t]{2}{*}{24.} & 7018 & Manadhar, N.P. & Stellaria lanata Hook. F. ex Edgew & \\
\hline & & & \& Hook.f. & Caryophylaceae \\
\hline 25. & 8010 & Manadhar, N.P. & Cerastium fontanum Baumg & Caryophylaceae \\
\hline 26. & 0121 & Basnet, B.K. & Euonymus amygdalifolius Franch. & Celastraceae \\
\hline 27. & 0126 & Basnet, B.K. & Euonymus sanguineus Loes.ex Diels. & Celastraceae \\
\hline 28. & 0220 & Basnet, B.K. & Euonymus fimbriatus Wall. & Celastraceae \\
\hline 29. & 0013 & Basnet, B.K. & Gnaphalium affine D.Don & Compositae \\
\hline 30. & 0010 & Basnet, B.K. & Gnaphalium bypoleucum DC. & Compositae \\
\hline 31. & 0085 & Basnet, B.K. & Ligularia fischeri (Ledeb.) Turcz. & Compositae \\
\hline 32. & 0092 & Basnet, B.K. & Morina polyphylla Wall. & Compositae \\
\hline 33. & 0117 & Basnet, B.K. & $\begin{array}{l}\text { Anaphalis busua (Buch. - Ham. } \\
\text { ex D.Don) DC. }\end{array}$ & Compositae \\
\hline 34. & 0124 & Basnet, B.K. & Conyza stricta Wall. & Compositae \\
\hline 35. & 0133 & Basnet, B.K. & $\begin{array}{l}\text { Conyza leucantha (D.don) Ludlow } \\
\text { \& Raven }\end{array}$ & Compositae \\
\hline 36. & 0134 & Basnet, B.K. & Asterpeduncularis Wall. Ex Nees & Compositae \\
\hline 37. & 0135 & Basnet, B.K. & $\begin{array}{l}\text { Cremanthodium arnicoides } \\
\text { (DC. Ex Royle) R. Good }\end{array}$ & Compositae \\
\hline
\end{tabular}




\begin{tabular}{|c|c|c|c|c|}
\hline \multirow{3}{*}{$\begin{array}{l}38 . \\
39 .\end{array}$} & 0156 & Basnet, B.K. & Dubyaea hispida DC. & Compositae \\
\hline & 0157 & Basnet, B.K. & Leontopodium stracheyi (Hook.f.) & \\
\hline & & & C.B. Clarke ex Hemsley & Compositae \\
\hline 40. & 0177 & Basnet, B.K. & Dipsacus inermis Wall & Compositae \\
\hline 41. & 0178 & Basnet, B.K. & Crassocephalum crepidioides (Benth.) & \\
\hline & & & S. Moore & Compositae \\
\hline 42. & 7038 & Manadhar, N.P. & Cirsium verutum (D.Don) Spreng & Compositae \\
\hline 43. & 8143 & Manadhar, N.P. & Carpesium nupalense Less. & Compositae \\
\hline 44. & P0, P013 & Basnet, B.K. & Erigeron acer Linn & Compositae \\
\hline 45. & P001 & Basnet, B.K. & Taraxacum officinale Wigg. & Compositae \\
\hline 46. & P002 & Basnet, B.K. & Artemisia carnifolia Buch.-Ham. & Compositae \\
\hline 47. & P010 & Basnet, B.K. & Conyza canadensis (L.) Cornq. & Compositae \\
\hline 48. & P034 & Basnet, B.K. & Myriactis nepalensis Less & Compositae \\
\hline 49. & P18 & Basnet, B.K. & Conyza japonica (Thunb.) Less. Ex Dc. & Compositae \\
\hline 50. & 0011 & Basnet, B.K. & Coriaria napalensis Wall. & Coriariaceae \\
\hline 51. & 0012 & Basnet, B.K. & Capsella bursa-pastoris (L.) Medikus & Cruciferae \\
\hline 52. & 0166 & Basnet, B.K. & Cardamine impatiens Linn. & Cruciferae \\
\hline 53. & 0181 & Basnet, B.K. & Cardamine macrophylla Willd. & Cruciferae \\
\hline 54. & 0194 & Basnet, B.K. & Cardamine loxostemonoids O.E. Schulz & Cruciferae \\
\hline 55. & P015 & Basnet, B.K. & Thlaspi arvense Linn. & Cruciferae \\
\hline 56. & 206712 & Basnet, B.K. & Juniperus indica Bertol. & Cupressaceae \\
\hline 57. & 0089 & Basnet, B.K. & Anthoxanthum hookeri (Griseb.) Rendle & Cyperaceae \\
\hline 58. & 0122 & Basnet, B.K. & Cyperus squarrosus L. & Cyperaceae \\
\hline 59. & 0127 & Basnet, B.K. & Carex birtella Drejer & Cyperaceae \\
\hline 60. & 0130 & Basnet, B.K. & Cyperus cyperoides (Retz.) Kuntze & Cyperaceae \\
\hline 61. & 0138 & Basnet, B.K. & Arundo donax Linn. & Cyperaceae \\
\hline 62. & 0139 & Basnet, B.K. & Cyperus difformis L. & Cyperaceae \\
\hline 63. & 0145 & Basnet, B.K. & Cyperus niveus Retz. & Cyperaceae \\
\hline 64. & 0159 & Basnet, B.K. & Eleocharis congesta D.don & Cyperaceae \\
\hline 65. & 0169 & Basnet, B.K. & Carex inanis Kunth & Cyperaceae \\
\hline 66. & 0173 & Basnet, B.K. & Isolepis setacea (L.) R.Br & Cyperaceae \\
\hline 67. & 0188 & Basnet, B.K. & Fimbristylis complanata (Retz.) Link & Cyperaceae \\
\hline 68. & 0192 & Basnet, B.K. & Schoenoplectus juncoides (Roxb.)Palla & Cyperaceae \\
\hline 69. & P027 & Basnet, B.K. & Arundinella setosa Trin. & Cyperaceae \\
\hline 70. & 0191 & Basnet, B.K. & Dioscorea deltoidea Wall. Ex Kunth & Dioscoreaceae \\
\hline 71. & 0171 & Basnet, B.K. & Dipsacus inermis Wall. & Dipsacaceae \\
\hline 72. & 0182 & Basnet, B.K. & Eleagnus kanaii Momiyama & Elaeagnaceae \\
\hline 73. & P009 & Basnet, B.K. & Eleagnus parvifolia Wall. & Elaeagnaceae \\
\hline 74. & 0014 & Basnet, B.K. & Rhododendron arboreum Smith & Ericaceae \\
\hline 75. & 0094 & Basnet, B.K. & Lyonia ovalifolia (wall.) Drude & Ericaceae \\
\hline 76. & 0176 & Basnet, B.K. & Gaultheria trichophylla Royle & Ericaceae \\
\hline 77. & 0015 & Basnet, B.K. & Rhododendron lepidotum Wall. Ex G.Don & Ericaeae \\
\hline 78. & 0105 & Basnet, B.K. & Quercus semicarpifolia $\mathrm{Sm}$. & Fagaceae \\
\hline 79. & 0107 & Basnet, B.K. & Quercus griffithi Hook.f. \& Thomas. ex Miq. & Fagaceae \\
\hline 80. & 0057 & Basnet, B.K. & Corydalis elegans Wall.ex Hook.f. \&Thomas & Fumariaceae \\
\hline 81. & 0059 & Basnet, B.K. & Corydalis govaniana Wall & Fumariaceae \\
\hline 82. & 0016 & Basnet, B.K. & Gentiana pedicellata (D.Don) Griseb. & Gentianaceae \\
\hline 83. & 0017 & Basnet, B.K. & Swertia bimaculata (Sieb. \& Zucc.) C.b. Clarke & Gentianaceae \\
\hline 84. & 0165 & Basnet, B.K. & Swertia nervosa (G.Don) C.B. Clarke & Gentianaceae \\
\hline 85. & P13 & Basnet, B.K. & Swertia chirayita (Roxb. ex Fleming) Karstrn & Gentianaceae \\
\hline 86. & 0190 & Basnet, B.K. & Geranium himalayanse Klotzsch & Geraniaceae \\
\hline 87. & 8091 & Basnet, B.K. & Geranium wallichianum D.Don ex Sweet & Geraniaceae \\
\hline 88. & 6997 & Manadhar, N.P. & Geranium lambertii Sweet & Geraniaceae \\
\hline 89. & 0074 & Basnet, B.K. & Microstegium petiolare (Trin.) Bor. & Gramineae \\
\hline 90. & 0080 & Basnet, B.K. & Oryzopsis lateralis (Regel) Stapf & Gramineae \\
\hline 91. & 0116 & Basnet, B.K. & Cappilipedium parviflorum (R.Br.) Stapf. & Gramineae \\
\hline
\end{tabular}




\begin{tabular}{|c|c|c|c|c|}
\hline 92. & 0119 & Basnet, B.K. & Agrostis pilosula Trin & Gramineae \\
\hline 93. & 0120 & Basnet, B.K. & Agrostis stolonifera Linn & Gramineae \\
\hline 94. & 0136 & Basnet, B.K. & Elymus nayarii Karthik & Gramineae \\
\hline 95. & 0137 & Basnet, B.K. & Brachypodium sylvaticum (Huds.) P. Beauv. & Gramineae \\
\hline 96. & 0140 & Basnet, B.K. & Agrostis petelotii (Hitchc.) Noltie & Gramineae \\
\hline 97. & 0163 & Basnet, B.K. & Themeda hookeri (Griseb.) A. Camus. & Gramineae \\
\hline 98. & 0174 & Basnet, B.K. & Heteropogon contortus (1.) Beauv. & Gramineae \\
\hline 99. & 0189 & Basnet, B.K. & Eragrostis nigra Nees \& Steud. & Gramineae \\
\hline 100. & 0193 & Basnet, B.K. & Cymbopogon munroi (C.B. Clarke) Noltie & Gramineae \\
\hline 101. & 0195 & Basnet, B.K. & Agrostis nervosa Nees ex Trin. & Gramineae \\
\hline 102. & 0224 & Basnet, B.K. & Chrysopogon gryllus (L.)Trin. & Gramineae \\
\hline 103. & 0018 & Basnet, B.K. & Myriophylum spicatum Linn. & Haloragacae \\
\hline 104. & 7062 & Manandhar, N.P & Hypericum choisianum Wall. Ex N. Robson & Hypericaceae \\
\hline 105. & 0184 & Basnet, B.K. & Hypericum elodeoides Choisy & Hypericaeae \\
\hline 106. & 0019 & Basnet, B.K. & Iris goniocarpa Baker & Iridaceae \\
\hline 107. & 0072 & Basnet, B.K. & Iris kemaonensis D.Don ex Royle & Iridaceae \\
\hline 108. & 0179 & Basnet, B.K. & Iris decora Wall. & Iridaceae \\
\hline 109. & 0148 & Basnet, B.K. & Juglans regia Linn. & Juglandaceae \\
\hline 110. & 0076 & Basnet, B.K. & Luzula muetiflora (Retz.) Lejeune & Juncaceae \\
\hline 111. & 0141 & Basnet, B.K. & Juncus leucanthus Royle ex D.Don & Juncaceae \\
\hline 112. & 0020, P025 & Basnet, B.K. & Thymus linearis Benth. & Labiatae \\
\hline 113. & 0021 & Basnet, B.K. & Nepeta laevigata (D.Don) Hand.-Mazz. & Labiatae \\
\hline 114. & 0022 & Basnet, B.K. & Verbascum Thapsus Linn. & Labiatae \\
\hline 115. & 0073 & Basnet, B.K. & Clinopodium umbrosum (M.Bieb.) C. Koch & Labiatae \\
\hline 116. & 0096 & Basnet, B.K. & Phlomis setigera Falc. ex Benth. & Labiatae \\
\hline 117. & 0155 & Basnet, B.K. & Leonurus cardiaca Linn. & Labiatae \\
\hline 118. & 0175 & Basnet, B.K. & Coleus forskoblii Briq. & Labiatae \\
\hline 119. & 0200 & Basnet, B.K. & Salvia nubicola Wall. & Labiatae \\
\hline 120. & 206760 & Basnet, B.K. & Dracocephalum wallichii Sealy & Labiatae \\
\hline 121. & 206787 & Basnet, B.K. & Colquhounia coccinea Wall & Labiatae \\
\hline 122. & 5156 & Manadhar, N.P. & Phlomis bracteosa Royle ex Benth. & Labiatae \\
\hline 123. & 7049 & Manadhar, N.P. & Elsholtzia fruticosa (D.Don0 Rehder & Labiatae \\
\hline 124. & 8577 & Manadhar, N.P. & Elsholtria blanda Benth. & Labiatae \\
\hline 125. & P003 & Basnet, B.K. & Salvia campanulata Wall. & Labiatae \\
\hline 126. & P12 & Basnet, B.K. & Origanum vulgare Linn. & Labiatae \\
\hline 127. & P4 & Basnet, B.K. & Salvia bians Royle ex Benth. & Labiatae \\
\hline 128. & 0023 & Basnet, B.K. & Caragana gerardiana Marquand & Leguminosae \\
\hline 129. & 0024 & Basnet, B.K. & Astragalus melanostachys Benth.ex Bunge & Leguminosae \\
\hline 130. & 0025 & Basnet, B.K. & Latbyrus luteus & Leguminosae \\
\hline 131. & 0026 & Basnet, B.K. & Trigonella emodi Benth. & Leguminosae \\
\hline 132. & 6923 & Manadhar, N.P. & Caragana brevispina Royle & Leguminosae \\
\hline 133. & 7010 & Manadhar, N.P. & Vicia angustifolia L. & Leguminosae \\
\hline 134. & 8136 & Manadhar, N.P. & Indigofera hebepetala Benth. ex Baker & Leguminosae \\
\hline 135. & P019 & Basnet, B.K. & Lotus corniculatus L. & Leguminosae \\
\hline 136. & 0147 & Basnet, B.K. & Urticularia australis R.Br. & Lentibulariaceae \\
\hline 137. & 0167 & Basnet, B.K. & Polygonatum verticillatum L. All. & Liliaceae \\
\hline 138. & 0183 & Basnet, B.K. & Smilax menispermoidea A.DC. & Liliaceae \\
\hline 139. & 0186 & Basnet, B.K. & Smilacina purpurea Wall. & Liliaceae \\
\hline 140. & 0199 & Basnet, B.K. & Scurrula elata (edgew.) Danser & Loranthaceae \\
\hline 141. & 0027 & Basnet, B.K. & Menyanthes trifolita Linn. & Menyanthaceae \\
\hline 142. & 0028 & Basnet, B.K. & Syringa emodi Wall. & Oleaceae \\
\hline 143. & 0029 & Basnet, B.K. & Jasminum bumile Linn. & Oleaceae. \\
\hline 144. & $\mathrm{P} 017 / 2$ & Basnet, B.K. & Oenothera rosea L.Herit \& Aiton & Onagraceae \\
\hline 145. & 0172 & Basnet, B.K. & Epipactis helleborine (L.) Crantz & Orchidaceae \\
\hline 146. & 0031 & Basnet, B.K. & Oxalis cornuculata Linn & Oxalidacae \\
\hline 147. & 0063 & Basnet, B.K. & Meconopsis regia $\mathrm{G}$. Taylor & Papaveraceae \\
\hline 148. & 0067 & Basnet, B.K. & Meconopsis napaulensis DC & Papaveraceae \\
\hline
\end{tabular}




\begin{tabular}{|c|c|c|c|c|}
\hline 149. & 0032 & Basnet, B.K. & Phytolaca acinosa Roxb. & Phytolacaceae \\
\hline 150. & 0008 & Basnet, B.K. & Pinus wallichiana A.B. Jackson & Pinaceae \\
\hline 151. & 0078 & Basnet, B.K. & Picea smithiana (Wall.)Boiss. & Pinaceae \\
\hline 152. & 0158 & Basnet, B.K. & Tsuga dumosa (D.Don) Eichler & Pinaceae \\
\hline 153. & 0170 & Basnet, B.K. & Cedrus deodara (Roxb. ex D.Don) G.Don & Pinaceae \\
\hline 154. & 0180 & Basnet, B.K. & Abiespindrow Royle & Pinaceae \\
\hline 155. & 0034 & Basnet, B.K. & Plantago major Linn. & Plantaginaceae \\
\hline 156. & 0088 & Basnet, B.K. & Polygala sibirica Linn. & Polygalaceae \\
\hline 157. & P017 & Basnet, B.K. & Polygala abyssinica R.Br. ex Fresen & Polygalaceae \\
\hline 158. & P035 & Basnet, B.K. & Polygonum aviculare Linn. & Polygonacae \\
\hline 159. & 0009 & Basnet, B.K. & Oxyria digyna (L.) Hill & Polygonaceae \\
\hline 160. & 0164 & Basnet, B.K. & Bistorta amplexicaulis (D.Don) Greene & Polygonaceae \\
\hline 161. & 0201 & Basnet, B.K. & Rumex acetosa Linn. & Polygonaceae \\
\hline 162. & 0035 & Basnet, B.K. & Potamogeton natans Linn. & Potamogetonaceae \\
\hline 163. & 0036 & Basnet, B.K. & Potamegeton pusillus Linn. & Potamogetonaceae \\
\hline 164. & 0102 & Basnet, B.K. & Potamogeton distintus & Potamogetonaceae \\
\hline 165. & P032 & Basnet, B.K. & Primula denticulata $\mathrm{Sm}$. & Primulaceae \\
\hline 166. & 0129 & Basnet, B.K. & Anemone rivularis Buch.-Ham. ex DC & Ranunculaceae \\
\hline 167. & 0161 & Basnet, B.K. & Thalictrum javanicum $\mathrm{Bl}$. & Ranunculaceae \\
\hline 168. & 0162 & Basnet, B.K. & Thalictrum virgatum Hook. f. \& Thoms & Ranunculaceae \\
\hline 169. & P015 & Basnet, B.K. & Thalictrum chelidonii DC & Ranunculaceae \\
\hline 170. & P037 & Basnet, B.K. & Caltha palustris Linn. & Ranunculaceae \\
\hline 171. & P21 & Basnet, B.K. & Clematis connata DC & Ranunculaceae \\
\hline 172. & 0007 & Basnet, B.K. & Sorbaria tomentosa (Lindl.) Rhed. & Rosaceae \\
\hline 173. & 0001 & Basnet, B.K. & Rosa macrophylla Lindl. & Rosaceae \\
\hline 174. & 0040 & Basnet, B.K. & Potentilla argyrophylla Wall. ex Lehm. & Rosaceae \\
\hline 175. & 0041 & Basnet, B.K. & Rubus foliolosus D.Don & Rosaceae \\
\hline 176. & 0043 & Basnet, B.K. & Potentilla nepalensis Wall. ex Hook & Rosaceae \\
\hline 177. & 0060 & Basnet, B.K. & Cotoneaster nitidus Jacques & Rosaceae \\
\hline 178. & 0100 & Basnet, B.K. & Prunus domestica & Rosaceae \\
\hline 179. & 0103 & Basnet, B.K. & Rosa brunonii Lindl. & Rosaceae \\
\hline 180. & 0106 & Basnet, B.K. & Pyrus pashia Buch.-Ham. ex D.Don & Rosaceae \\
\hline 181. & 0132 & Basnet, B.K. & Prunus cornuta (Wall. ex Royale) Steud. & Rosaceae \\
\hline 182. & A2/P004 & Basnet, B.K. & Potentilla griffithii Hook. f. & Rosaceae \\
\hline 183. & P004 & Basnet, B.K. & Agrimonia pilosa Ledeb. & Rosaceae \\
\hline 184. & P007 & Basnet, B.K. & Cotoneaster tibeticus Klotz & Rosaceae \\
\hline 185. & P014/0094 & Basnet, B.K. & Cotoneaster microphyllus Wall. ex Lindl. & Rosaceae \\
\hline 186. & P020/P011 & Basnet, B.K. & Fragaria nubicola Lindle. ex Lacaita & Rosaceae \\
\hline 187. & P021 & Basnet, B.K. & Rosa sericea Lindl. & Rosaceae \\
\hline 188. & P029 & Basnet, B.K. & Cotoneaster taylori $\mathrm{Yu}$ & Rosaceae \\
\hline 189. & P030 & Basnet, B.K. & Potentilla leuconota D.don & Rosaceae \\
\hline 190. & P11 & Basnet, B.K. & Spiraea bypoleuca Dunn & Rosaceae \\
\hline 191. & P0 52 & Basnet, B.K. & Rubia manjith Roxb. ex Fleming & Rubiaceae \\
\hline 192. & 0044 & Basnet, B.K. & Skimmia anquetilia N.P. Tylor \& Airy Shaw & Rutaceae \\
\hline 193. & 0045 & Basnet, B.K. & Populus ciliata Wall. ex Royle & Salicaceae \\
\hline 194. & 0046 & Basnet, B.K. & Salix daltoniana Andress. & Salicaceae \\
\hline 195. & 2067103 & Basnet, B.K. & Viburnum cotininfolium D.Don & Sambucaceae \\
\hline 196. & 206719 & Basnet, B.K. & Viburnum erubescens Wall. ex DC. & Sambucaceae \\
\hline 197. & 0093 & Basnet, B.K. & Bergenia ciliata (Haw.) Sternb. & Saxifragaceae \\
\hline 198. & 0095 & Basnet, B.K. & Saxifraga parnassifolia D.Don. & Saxifragaceae \\
\hline 199. & 0097 & Basnet, B.K. & Astilbe rivularis Buch.-Ham. ex D.Don & Saxifragaceae \\
\hline 200. & 0098 & Basnet, B.K. & Parnacea nubicola Wall. Ex Royale & Saxifragaceae \\
\hline 201. & 0079 & Basnet, B.K. & Sopubia trifida Buch.- Ham. Ex D.Don & Scrophulariaceae \\
\hline 202. & 0101 & Basnet, B.K. & Hemiphragma heterophyllum Wall. & Scrophulariaceae \\
\hline 203. & 0108 & Basnet, B.K. & Mazus surculosus D.don & Scrophulariaceae \\
\hline 204. & 0109 & Basnet, B.K. & Peducularis gracilis Wall. Ex Benth. & Scrophulariaceae \\
\hline 205. & 0146 & Basnet, B.K. & Verbascum thapsus $\mathrm{L}$. & Scrophulariaceae \\
\hline
\end{tabular}




\begin{tabular}{|c|c|c|c|c|}
\hline 206. & 0083 & Basnet, B.K. & Datura stramonium Linn. & Solanaceae \\
\hline 207. & 0091 & Basnet, B.K. & Hyosymus niger Linn. & Solanaceae \\
\hline 208. & 0049 & Basnet, B.K. & Myricaria rosea W.W.Sm. & Tamaricaceae \\
\hline 209. & 0048 & Basnet, B.K. & Taxus contorta Griff & Taxaceae \\
\hline 210. & $\mathrm{P} 019 / 2$ & Basnet, B.K. & Stellera chamaejasme Linn. & Thymelaceae \\
\hline 211. & 0065 & Basnet, B.K. & Torilis japonica (Houtt.) DC. & Umbelliferae \\
\hline 212. & 0070 & Basnet, B.K. & Pleurospermum hookeri C.B. Clarke & Umbelliferae \\
\hline 213. & 0077 & Basnet, B.K. & Bupleurum longicaule Wall. ex DC. & Umbelliferae \\
\hline 214. & 0081 & Basnet, B.K. & Acronema tenerum (DC.) Edgew. & Umbelliferae \\
\hline 215. & 0082 & Basnet, B.K. & Bupleurum tenue Wall. ex DC & Umbelliferae \\
\hline 216. & 0084 & Basnet, B.K. & Heracleum nepalense D.Don & Umbelliferae \\
\hline 217. & 8561 & Manadhar, N.P. & Bupleurum candoleii Wall. ex DC. & Umbelliferae \\
\hline 218. & 8775 & Manadhar, N.P. & Pimpinella diversifolia DC. & Umbelliferae \\
\hline 219. & P024 & Basnet, B.K. & Eryngium foetidum Linn. & Umbelliferae \\
\hline 220. & 0075 & Basnet, B.K. & Urtica dioca Linn. & Urticaceae \\
\hline 221. & 0058 & Basnet, B.K. & Dipsacus inermis Wall. & Violaceae \\
\hline 222. & 0062 & Basnet, B.K. & Viola biflora Linn. & Violaceae \\
\hline 223. & 0071 & Basnet, B.K. & Parthenocissus semicordata (Wall) Planch. & Vitaceae \\
\hline 224. & 0052 & Basnet, B.K. & Roscoea alpina Royle & Zingiberaceae \\
\hline
\end{tabular}

